\title{
NANOTECNOLOGÍA: GESTIÓN Y REGLAMENTACIÓN DE RIESGOS PARA LA SALUD Y MEDIO AMBIENTE EN AMÉRICA LATINA Y EL CARIBE
}

\author{
NANOTECHNOLOGY: RISK MANAGEMENT AND REGULATION FOR HEALTH AND ENVIRONMENT IN \\ LATIN AMERICA AND IN THE CARIBBEAN
}

\author{
Guillermo Foladori $^{1}$ \\ Fernando Bejarano $^{2}$ \\ Noela Invernizzi ${ }^{3}$
}

Resumo Este artículo analiza la discusión sobre los riesgos de las nanopartículas manufacturadas llevada a cabo en las reuniones regionales de América Latina y el Caribe del SAICM (Strategic Approach to International Chemicals Management/Enfoque Estratégico para la Gestión de Productos Químicos a Nivel Internacional). Contextualiza esta discusión con un panorama del desarrollo de las nanotecnologías en la región y de las evidencias científicas disponibles sobre riesgos de las nanotecnologías. Propone un abordaje para identificar a los actores que deben participar en la discusión y gestión del riesgo basado en el ciclo de vida de las nanopartículas. El artículo propone, además, algunas condiciones necesarias para incentivar un desarrollo responsable de las nanotecnologías desde ámbitos como el SAICM, y trae a la discusión los ocho puntos relevados por más de cien organizaciones ambientalistas y de trabajadores acerca de la supervisión de las nanotecnologías y los nanomateriales.

Palavras-chave nanotecnologías; SAICM; riesgos; trabajadores; consumidores.
Abstract This article addresses the discussion concerning the risks posed by manufactured nanoparticles held during regional meetings in Latin America and the Caribbean by the SAICM (Strategic Approach to International Chemicals Management/Enfoque Estratégico para la Gestión de Productos Químicos a Nivel Internacional). It contextualizes this discussion by overviewing the development of nanotechnology in the region and the scientific evidence available on the risks brought about by nanotechnology. It proposes an approach to identify the players that should be involved in the discussion and in risk management based on the nanoparticles' life cycle. The article also proposes a few of the conditions needed to encourage the responsible development of nanotechnology from sectors such as the SAICM, and brings into the discussion the eight relevant items from over a hundred environmental organizations and workers under the supervision of nanotechnologies and nanomaterials. Keywords nanotechnologies; SAICM; risks; workers; consumers. 


\section{Introducción}

Liderada por los países más industrializados y China, la investigación en nanotecnologías (NT) se ha intensificado decisivamente a partir del inicio del siglo. Consideradas la base de una nueva revolución tecnológica, estas tecnologías son objeto de grandes inversiones públicas y privadas en investigación y desarrollo (I\&D) y están comenzando a ser incorporadas en prácticamente todos los sectores industriales. Un considerable número de países en desarrollo, incluyendo los de América Latina y el Caribe, han incorporando las NT en sus políticas de ciencia, tecnología e innovación desde mediados de la década pasada.

El surgimiento de crecientes evidencias científicas sobre riesgos de las nanopartículas y materiales nanofacturados para la salud humana y el ambiente ha generado discusiones y temores en varios sectores de la sociedad civil. Científicos, órganos reguladores, empresarios y grupos organizados de la sociedad han manifestado posiciones diversas sobre el tema, y la cuestión de la gobernanza y regulación de las nanotecnologías ha adquirido creciente interés a nivel global.

El “Enfoque Estratégico para la Gestión de Productos Químicos a Nivel Internacional", mejor conocido como SAICM (Strategic Approach to International Chemicals Management), ha iniciado discusiones sobre los riesgos de las NT y está elaborando propuestas de acción para reducirlos y prevenirlos. Se trata de un acuerdo voluntario, destinado a proteger la salud humana y el medio ambiente, que busca regular las sustancias químicas en todo el ciclo de vida de los productos, incluyendo aspectos ambientales, de salud ocupacional y de salud pública. El SAICM resulta un ámbito estratégico por ser el único espacio institucional multilateral en el seno de la Organización de las Naciones Unidas donde se están discutiendo los riesgos de las NT y por su carácter participativo, que envuelve representantes de los gobiernos, de organizaciones no gubernamentales tanto de la industria como de interés público, y organizaciones sindicales.

Este artículo da cuenta del estado de la discusión de las NT en el ámbito del SAICM, en América Latina y el Caribe. Contextualiza esa discusión en el avance del desarrollo de las NT en la región y en el surgimiento de crecientes evidencias sobre riesgos de las NT. Ofrece una reflexión sobre los riesgos a la salud y el medio ambiente del ciclo de vida de los nanomateriales, así como el lugar de los diferentes agentes sociales en dicho ciclo. Discute algunas condiciones para el desarrollo responsable de las NT y algunas propuestas que más de cien organizaciones a nivel global han elaborado como forma de evaluar y monitorear el desarrollo de estas nuevas tecnologías y sus productos.

El artículo se compone de cuatro apartados. En el primero se traza un breve panorama de las políticas y acciones en NT implementadas por varios 
países de la región. En el segundo se presentan las características de esta nueva tecnología y se muestra cómo en los últimos años ha aumentado la cantidad de evidencias científicas sobre riesgos de las NT. En la tercera, se explica el objetivo del SAICM y las propuestas de las resoluciones adoptadas por los países y participantes no gubernamentales de América Latina y el Caribe en las reuniones regionales realizadas en los años 2010 y 2011 . En el último apartado se analiza el ciclo de vida de los nanomateriales manufacturados, así como el nivel de riesgo asociado para los diferentes sectores sociales involucrados; y se exponen algunos conceptos claves de gobernanza de las NT.

\section{Las NT en América Latina y el Caribe}

Brasil, México y Argentina, los tres mayores países de la región y con capacidades científicas más consolidadas, fueron los primeros en estimular el desarrollo de las NT durante los primeros años del siglo. Rápidamente ocuparon las posiciones más destacadas en número de investigadores en NT, infraestructura de investigación y publicaciones científicas (Kay \& Shapira, 2009; OICTI, 2009).

En Brasil, el Ministerio de Ciencia y Tecnología (MCT) y sus agencias comenzaron a estimular el desarrollo de la nanotecnología a fines del año 2000. La primera acción fue el financiamiento de cuatro redes cooperativas de investigación básica y aplicada en nanociencia y NT con el propósito de consolidar el conocimiento en el área (Toma, 2005). En 2004, un programa para el desarrollo de las NT fue incorporado al plan Plurianual 2004-2007 de Ciencia y Tecnología, el cual fue ampliado un año más tarde con el lanzamiento del Programa Nacional de Nanotecnología. Éste financió actividades de I\&D, con particular atención a las asociaciones entre universidad y empresa, la construcción y renovación de laboratorios, proyectos de incubadoras de empresas de NT y la calificación de recursos humanos. Diez nuevas redes cooperativas fueron financiadas entre 2005 y 2009, y otras 17 lanzadas en 2010. Actualmente hay cerca de 50 universidades y centros de investigación con más de 1200 investigadores y 2000 estudiantes universitarios trabajando en las diversas áreas de NT. De los 120 Institutos Nacionales de Ciencia y Tecnología creados por el MCT a fines del 2008, 21 realizan investigaciones en NT. El país cuenta con unas 150 empresas que desarrollan o aplican NT (Invernizzi et al., 2011). El MCT invirtió desde la formulación del primer programa de nanotecnología, en 2004, hasta 2009, un valor próximo a 314 millones de reales (en torno a 190 millones de dólares al cambio de marzo de 2010)4 (MCT, 2008; Embrapa Notícias, 2009).

En México, el Programa Especial de Ciencia y Tecnología 2001-2006 (PECyT), incluido en el Plan Nacional de Desarrollo, colocó las NT como 
área estratégica de desarrollo dentro del departamento de materiales avanzados. Dicho programa subrayó la necesidad de crear un Programa Nacional de Nanotecnología y una red científica en el área (CONACYT, 2002). El primero aún no se concretó y la Red de Nanociencia y Nanotecnología fue creada en 2009. Según estimaciones, entre 1998 y 2004, 14.4 millones de dólares de fondos públicos fueron destinados a I\&D en NT (Cimav, 2008). Para 2010 la implementación de la política de NT había tomado cuerpo en al menos tres desarrollos importantes: la creación de la Red de Nanociencia y Nanotecnología (2009), la construcción de dos Laboratorios nacionales (2007), y el desarrollo de parques de ciencia y tecnología donde gobierno, empresas y academia se integran. En el Estado de Nuevo León, un Cluster de Nanotecnología fue creado con una incubadora de empresas de NT orientadas principalmente para las demandas de la industria cosmética. En 2010 había más de 60 universidades y centros de investigación con proyectos en NT y en torno de 500 investigadores incorporados. El número de empresas en el área se estima en 70 (Foladori \& Zayago, 2011).

En Argentina, la Secretaría de Ciencia y Tecnología estableció las NT como una de las áreas prioritarias de financiamiento a partir de 2003. Un seminario sobre el tema fue organizado en 2004, y en el mismo año la Agencia Nacional de Promoción de la Investigación Científica y Tecnológica (ANPCyT) incluyó las NT en un llamado para la presentación de proyectos de investigación (García et al., 2011). En 2005 fue creada la Fundación Argentina de Nanotecnología (FAN) con un presupuesto federal de 10 millones de dólares para los siguientes 5 años. La ANPCyT, a través de su fondo sectorial, comenzó una nueva línea de financiamiento en tres áreas de NT en 2010: nanomateriales, nanointermediarios y nanosensores, con un presupuesto de 10 millones de dólares orientados a la formación de asociaciones público-privadas (FONARSEC, 2010). Cuatro redes, con cerca de 200 investigadores, abordan las áreas de nanotecnología molecular, nanomateriales, Mems/Nems (micro/nano-electromechanical systems), y bio-materiales. Además, hay algu-nos grupos trabajando en nanomedicina. Se han identificado 25 empresas con actividades en nanotecnología (Garcia et al., 2011; Spivack et al., 2011).

Países de mediano tamaño, como Colombia, Chile y Venezuela también vienen estimulando el desarrollo de las NT. En 2004, el secretariado de Ciencia y Tecnología de Colombia (Conciencias) colocó las NT dentro de las ocho áreas consideradas estratégicas para el país (Medina, 2005); y en 2005 se estableció el Consejo Nacional de Nanociencia y Nanotecnología, asignado a la sección colombiana del Instituto de Ingeniería Eléctrica y Electrónica (IEEE). El país tiene 19 grupos de investigación en diez universidades y una red de desarrollo de investigación en Nanotecnociencia (CNNN, 2005). Chile tiene varios grupos de investigación en sus principales universidades. El Minis- 
terio de Educación, a través de la agencia de ciencia y tecnología CONICYT, y el Ministerio de Economía han financiado la investigación en NT en cuatro centros: el Centro de Nanociencias de Valparaiso, el Centro para el Desarrollo de la Nanociencia y Nanotecnología, el Centro Interdisciplinario para Iones Líquidos, y el Centro de Magnetismo Básico y Aplicado (Cortez, 2011). El Plan Nacional de Ciencia, Tecnología e Innovación 2005-2030 de Venezuela resaltó la importancia de desarrollar tecnologías de vanguardia, incluyendo las NT. En 2010 se creó la Red Venezolana de Nanotecnología, la cual congregó a investigadores de las principales universidades y centros de investigación, así como a representantes del sector productivo y de agencias gubernamentales (López et al., 2011). En Perú, el Plan Estratégico Nacional de Ciencia, Tecnología e Innovación para la Competitividad y el Desarrollo Humano (SINACyT/CONCYTEC, 2005) colocó las NT como un área estratégica para el desarrollo del país. El número de investigadores en el área está en torno de 25 (Gutarra, 2008).

Las actividades de NT también se han hecho presentes en los pequeños países de la región. En Uruguay, investigadores en el área se reúnen en el grupo NanotecUy, desde 2006. En 2010 las NT fueron incluidas como un área transversal prioritaria en el Plan Estratégico Nacional de Ciencia, Tecnología e Innovación (Chiancone, 2011). En la República Dominicana, el Plan Estratégico de Ciencia Tecnología e Innovación 2008-2018 incluyó las NT como área prioritaria dentro de las ciencias físicas. La investigación está concentrada en una universidad privada (Piazza, 2011). Costa Rica tiene un laboratorio de NT desde 2004 (Lanotec) y algunas universidades públicas tienen programas de investigación en NT, en particular en el área de polímeros y componentes electrónicos (Vega-Baudrit \& Campos, 2011). En Cuba, el Centro de Estudios Avanzados dedicado a las NT y las tecnologías convergentes fue lanzado en 2010. Las áreas de mayor desarrollo en Cuba están vinculadas a los materiales y las aplicaciones médicas y biotecnológicas (CubaDebate, 2010).

Este breve panorama muestra que las actividades de investigación en nanotecnología, así como su incorporación en procesos industriales han adquirido creciente dinamismo en los últimos años. Ello justifica la necesidad de atender los potenciales riesgos de estas tecnologías emergentes en el contexto regional.

\section{Riesgos a la salud y el medio ambiente de las nanotecnologías}

Las NT son diversas técnicas y métodos que permiten manipular la materia en escala atómica y molecular. Su novedad radica en por lo menos tres características de suma importancia para los procesos productivos. La primera 
es que, cuanto más reducido el tamaño de un material, mayor superficie externa posee en relación con su masa. Como la reactividad química de un material depende de su superficie externa, resulta que las nanopartículas tienen una reactividad química mucho mayor que los mismos materiales en una escala superior. Esto permite que una menor cantidad del producto pueda ofrecer iguales o mejores resultados en términos de combustión, oxidación, actividad biológica, etc.

La segunda característica es que cuando los materiales se reducen a tamaño nano - este tamaño no es fijo sino que depende de varias características y condiciones, pero por convención se estima de 1 a 100 nanómetros - sus propiedades físicas, químicas y toxicológicas cambian. Los nanotubos de carbono, por ejemplo, son 100 veces más rígidos que el acero y, al mismo tiempo, seis veces más livianos. El oro, que no es reactivo, se vuelve reactivo en nanoescala. El cobre, que es relativamente blando, se torna duro en nanoescala. Al explotar estas características se abre un nuevo umbral para aplicaciones industriales de diversos materiales.

La tercera característica de gran importancia para los procesos productivos es que al trabajar en escala nano se pueden realizar actividades que serían imposibles de otra manera, como, por ejemplo, incorporar nano sensores dentro de organismos vivos o en dispositivos de tamaños diminutos.

Las ventajas de trabajar en escala nano redundan en que se pueden crear nuevos materiales, en que los materiales conocidos pueden ofrecer nuevas propiedades o exhibir un desempeño más eficiente y en que un mismo producto puede tener múltiples funciones. Algunos ejemplos de ello son visibles en productos ya disponibles en el mercado. Las llantas para vehículos de la empresa Lanxess, por ejemplo, agregan a la materia prima nanopartículas de negro de carbón, haciéndolas más resistentes al desgaste y otorgándoles una vida útil, según dice la compañía, el 15\% mayor. El $\mathrm{DEPT}$, un material cerámico para remediación de efluentes en las industrias de papel y textil, manufacturado por la empresa Contech Productos Biodegradables, elimina el $95 \%$ del color de los efluentes, comparado con el $50 \%$ logrado por los métodos tradicionales de carbón activado. Además, es reciclable, y puede ser reutilizado varias veces. El té producido por Qinghuangdao Ialji Ring Nano-Product Co. Ltd contiene nanopartículas de selenio, agregándole a las propiedades del té las del selenio y haciéndolo así una bebida multifuncional. CHT Brasil Química produce textiles que transfieren vitamina E a la piel, convirtiendo a la vestimenta en un cosmético permanente. La LifeShirt es una camisa con nanosensores que monitorea la actividad respiratoria y cardíaca, al mismo tiempo que registra los datos y los almacena en sistemas portátiles que luego pueden interactuar con computadoras. Los automóviles que han sustituido la carrocería tradicional por una de 
polímeros, como algunos coches BMW, tienen la propiedad de conservar la memoria del momento de salida de la fábrica, de manera que ante abolladuras, pueden volver a su forma original aplicando corriente eléctrica. Frutas y nueces pueden ser recubiertas por filmes comestibles que las mantienen frescas por mucho más tiempo, como es el caso del nanofilm desarrollado por la empresa Embrapa para cubrir las nueces de macadamia. Estos y otros muchos ejemplos explican las ventajas competitivas de los productos de la nanotecnología y la razón por la cual están rápidamente ingresando al mercado.

Partículas de tamaño nano siempre han existido en la naturaleza, como en la ceniza volcánica, en el humo de incendios, en las nubes, en el polvo etc. También hay nanopartículas que son resultado secundario y/o involuntario de procesos de producción humanos, como las que aparecen en la combustión de motores, o en el asbesto o el cuarzo. Al hablar de nanotecnología no nos referimos a estos dos últimos tipos, sino a la producción voluntaria de nanopartículas. En inglés se utiliza el término nanoengineering o nano-ingeniería o nano fabricación o nano-manufactura para designar estas partículas que son creadas voluntaria y conscientemente por el ser humano para formar parte de procesos de producción.

Hasta hace unos años era común escuchar el argumento de que no había pruebas de que las partículas nano-manufacturadas ofrecían riesgo de toxicidad al ser humano y otros seres vivos. Efectivamente, durante la primera década del siglo XXI se produjo un crecimiento sostenido y amplio de diferentes tipos de nanopartículas, nanoestructuras y nanodispositivos que se fueron incorporando a productos comerciales en las más diversas ramas de la economía. Este rápido avance de la investigación y producción mercantil no estuvo acompañado de inversiones en cantidad suficiente que analizaran los riesgos de esos nuevos materiales, de manera que no había pruebas suficientes de su toxicidad. Sin embargo, poco a poco, se ha acumulado una gran cantidad de artículos científicos que sugieren que varias nanopartículas tienen propiedades tóxicas y, por lo tanto, requieren de un manejo adecuado.

El International Council on Nanotechnology (ICON), una institución de la Universidad de Rice (Estados Unidos) que investiga sobre riesgos de los nanomateriales, tiene un banco de información al respecto. De 2000 a 2010 este banco de datos registró un aumento sostenido de artículos publicados en revistas científicas arbitradas dedicados a analizar los potenciales riesgos de los nanomateriales en la salud humana y/o en el medio ambiente. La figura 1 ilustra en un gráfico este crecimiento. En 2010, que es el último año completo registrado, los artículos científicos publicados llegaron a 563. 
Figura 1

Evolución de la cantidad de artículos científicos arbitrados sobre riesgos a la salud y/o el medio ambiente de los nanomateriales manufacturados 2000-2010 - International Council on Nanotechnology

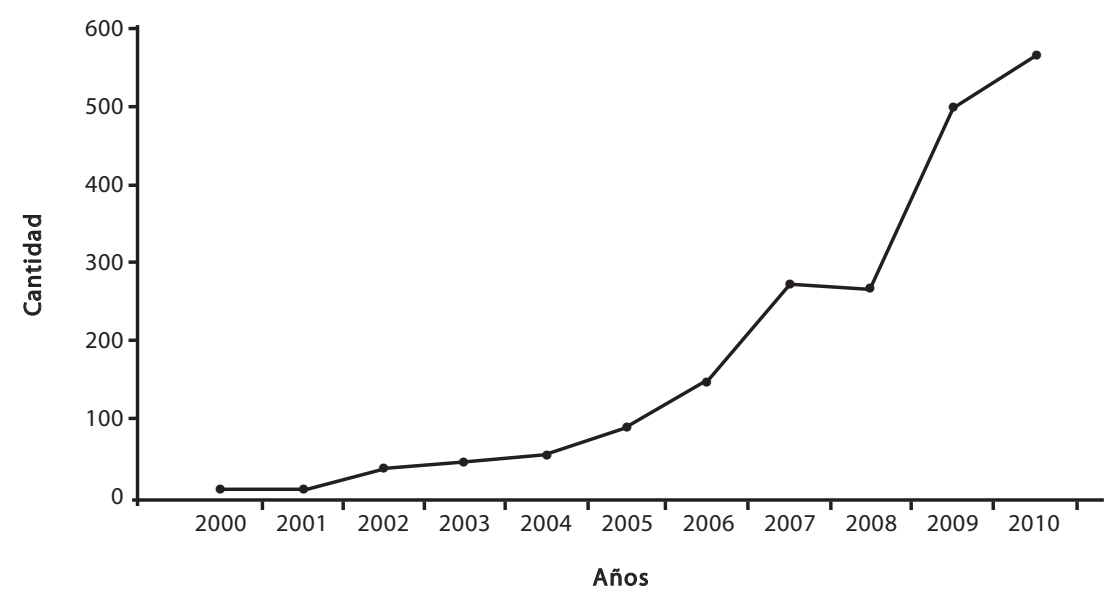

Fuente: Elaboración a partir del banco de datos de ICON (http://icon.rice.edu/) combinando los siguientes nanomateriales: [Carbon or Metal or Organic/Polymers or Semiconductor or Oxide or Multiple or Other/Unspecified] + Hazard para los siguientes grupos [Industrial/Research Worker or Consumers or General Population or Ecosystem or Other/Unspecified] + Peer Reviewed Journal Article + Engineered.

Otra organización, la NanoCeo (Nanotechnology Citizen Engagement Organization) elabora un banco de datos que permite clasificar los artículos científicos sobre riesgos de los nanomateriales según el tipo de material nanofacturado. Puede verse en la figura 2 que entre el año 2000 y finales de 2010 se han acumulado 176 artículos sobre riesgos de los nanotubos de carbono, 190 sobre riesgos de la nanoplata y 70 sobre riesgos del dióxido de titanio, entre otros materiales. Puede apreciarse cómo han ido aumentando los artículos científicos en la primera década del siglo, y, también, cómo algunos nano materiales sólo registran pocos artículos sobre sus potenciales riesgos. 
Figura 2

\begin{tabular}{|c|c|c|c|c|c|c|c|c|c|c|c|c|}
\hline Año & NTC & Fullerenos & Dendrimeros & $\begin{array}{l}\text { Partículas } \\
\text { ultrafinas } \\
\text { en el aire }\end{array}$ & $\begin{array}{c}\text { Quantum } \\
\text { dots/nano- } \\
\text { cristales }\end{array}$ & $\begin{array}{l}\text { Dióxido de } \\
\text { titanio }\end{array}$ & Plata & Zinc & Sílice & Oro & Cobre & Aluminio \\
\hline 2000 & & 1 & 2 & 2 & & 1 & 2 & & & & & \\
\hline 2001 & 1 & 1 & & 1 & & 3 & 2 & & & & & \\
\hline 2002 & & 3 & 2 & 7 & 1 & 3 & 2 & & & & & \\
\hline 2003 & 1 & 1 & 4 & 2 & 3 & 5 & 5 & & & & & \\
\hline 2004 & 5 & 3 & 3 & 1 & 5 & 2 & 9 & & & & & \\
\hline 2005 & 13 & 5 & 3 & 6 & 5 & 5 & 15 & & & & & 1 \\
\hline 2006 & 25 & 9 & 2 & 2 & 5 & 7 & 20 & 2 & 2 & & 1 & \\
\hline 2007 & 41 & 15 & 1 & 11 & 7 & 14 & 31 & 1 & 5 & 3 & 5 & 1 \\
\hline 2008 & 28 & 9 & & 1 & 9 & 17 & 36 & 2 & 1 & 2 & 1 & 1 \\
\hline 2009 & 8 & & & & 3 & 3 & 24 & 1 & 3 & 3 & 1 & \\
\hline 2010 & 54 & 6 & 2 & & 11 & 10 & 44 & 5 & 3 & 3 & 1 & 1 \\
\hline Total & 176 & 53 & 19 & 33 & 49 & 70 & 190 & 11 & 14 & 11 & 9 & 4 \\
\hline
\end{tabular}

Fuente: base de datos de NanoCeo (Nanotechnology Citizen Engagement Organization, disponible en: www.nanoceo.net/nanorisks; consultada en 22 nov. 2010).

La acumulación de información científica en estos y otros bancos de datos ya no permite ignorar la duda razonable de que varias nanopartículas son tóxicas para la salud humana y el medio ambiente. Sin embargo, el panorama resulta ser muy complicado. Hay nanopartículas de muy diferentes materiales, combinadas de distintas formas y potencialmente exponen a los organismos vivos de variadas maneras. Así que cada caso es un caso de toxicidad diferente, lo cual dificulta enormemente establecer normas y criterios de regulación.

Los estudios mencionados coinciden en que varias nanopartículas tienen un alto grado de toxicidad. Algunas de ellas se comportan de manera similar al asbesto. Hay evidencias, también, de que el tamaño de las nanopartículas es tan diminuto que cuando están presentes en el aire y son inhaladas, en lugar de ir a los pulmones, atraviesan directamente los conductos respiratorios, pasan la barrera de la sangre y llegan al cerebro. De igual forma se sabe que muchas de estas nanopartículas pueden atravesar la barrera que existe entre la madre y el feto, e inclusive, reaparecer en las futuras generaciones porque se biodegradan con gran lentitud. Las nanopartículas pueden, además, producir procesos electrónicos que hagan corto circuito en el metabolismo celular; pueden inclusive llegar a dañar y modificar el ADN. Su diminuto tamaño les permite entrar a las células e interactuar dentro de 
ellas. Es claro que muchas de estas comprobaciones se han realizado en pruebas in vitro en laboratorio, o en animales, y no hay pruebas estadísticas concluyentes de que lo mismo pueda suceder en los seres humanos. Además, prácticamente no hay estudios específicos que analicen los productos con nanocomponentes que están en el mercado, y que, en caso de conllevar riesgos, estarían ya exponiendo a la población y al medio ambiente. De manera que, a pesar de la creciente información disponible, lo que puede afirmarse es la existencia de duda razonable (Kulinowski, 2009; Maynard, 2006). Esta duda razonable debiera ser la base de una política de precaución; pero esta política aún no existe y el mercado sigue comandando el rumbo.

Oberdorster y colaboradores (2005) proclamaban la necesidad de una disciplina específica para encarar los potenciales riesgos de las nanopartículas, a la que llamaron nanotoxicología. Según ellos, son varios los parámetros que pueden implicar la toxicidad de las nanopartículas, y escriben:

La actividad biológica y bioquinética son dependientes de varios parámetros: tamaño, forma, química, cristalinidad, propiedades de superficie (área de porosidad, carga, modificaciones de superficie, desgaste de la capa superficial), grado de aglomeración, biopersistencia y dosis (Oberdorster et al., 2005, p. 823).

Un año más tarde, Maynard y colegas (2006) también advertían que las nanopartículas suponen una diferente toxicidad debido a sus propiedades; y mencionaban al menos seis características que pueden tener influencia en la toxicidad: el tamaño, el área de superficie, la química de superficie, la solubilidad, la forma y estructura, y la durabilidad en el tiempo. Estas características superan las normalmente utilizadas en los análisis de toxicidad de los materiales en escala mayor.

Diversas nanopartículas están hoy disponibles en el mercado en muy distintos productos. Hay nanopartículas de dióxido de titanio y de zinc en filtros solares, nanopartículas e iones de plata en refrigeradores, lavarropas, ropa de cama, ropa de vestir, envases de comida, etc. Hay nanotubos de carbono en pinturas, en aparatos de comunicación, etc. Hay dióxido de silicio en envoltorios de alimentos.

Se argumenta que la toxicidad que se puede demostrar in vitro no ocurre una vez que las mismas nanopartículas están incorporadas a productos de consumo final. En este sentido es importante distinguir las nanopartículas que están disueltas en líquidos, las que están en sólidos y aquellas incorporadas a matrices.

El Instituto Nacional para la Salud y Seguridad Ocupacional (NIOSH) de los Estados Unidos de América reconoce que los mayores riesgos de las nanopartículas están en polvos en estado sólido, dispersas o aglomeradas en 
los polvos, por ejemplo en cosméticos. En un segundo nivel de riesgo están las suspendidas en líquidos, como los nanotubos en agua. En un tercer nivel están las fijas en matrices, como los filmes delgados. Las que teóricamente ofrecerían menor riesgo son las incorporadas en nanoestructuras, como aleaciones en metales.

Esta escala de riesgo se asocia, también, a las diferentes maneras en que las nanopartículas pueden ingresar al organismo e interactuar con éste. En términos generales y no exclusivos, las principales vías de ingreso potencial de nanopartículas al organismo son la inhalación, la ingestión y la penetración a través de la piel. Cuando se trata de productos médicos con nanopartículas, ingresan vía inyección y por el desprendimiento de nanopartículas utilizadas en implantes (Maynard, 2006). Ciertamente, también es necesario tomar en cuenta el caso de accidentes, como incendios o explosiones, que pueden extender los riesgos de las nanopartículas a personas sin ningún tipo de protección.

Según el Departamento de Salud y Servicios Humanos del Centro para el Control y Prevención de las Enfermedades del NIOSH de los Estados Unidos de América, las nanopartículas pueden provocar inflamación, reubicación en determinados órganos, síntomas alérgicos similares al asma, síntomas de neumonía agravados y efectos cardíacos dos días después de la exposición, entre otros síntomas (DHHS, 2009).

\section{EL SAICM y las recomendaciones sobre nanotecnología de América Latina y el Caribe}

El SAICM, o “Enfoque Estratégico para la Gestión de Productos Químicos a Nivel Internacional" tiene como objetivo lograr la gestión racional de los productos químicos a través de todo su ciclo de vida, de manera que hacia el año 2020 "las sustancias químicas se produzcan y usen de manera que se reduzcan significativamente los impactos adversos sobre la salud y el medio ambiente" ${ }^{5}$ El SAICM está compuesto por una Declaración Política de alto nivel, una Estrategia de Política Global y un Plan Global de Acción (www.saicm.org). El SAICM fue adoptado formalmente por los ministros de medio ambiente y de salud de más de 100 países, con la participación activa de organismos de la industria química, de organizaciones de la sociedad civil de interés público y de sindicatos en la primera sesión de la Conferencia Internacional sobre la Gestión de los Productos Químicos (ICCM por su sigla en inglés) celebrada en Dubai, Emiratos Árabes Unidos“, en febrero del 2006 (Weinberg, 2008).

Las NT se propusieron como tema emergente a ser normado dentro del SAICM en la Segunda Conferencia de la ICCM celebrada en mayo del 2009 
en Ginebra, Suiza; sin embargo, el asunto tiene sus antecedentes en la llamada Declaración de Dakar durante la sexta reunión del Foro Intergubernamental de Seguridad Química (IFCS) de septiembre del 2008, donde se recomendaba que los gobiernos y la industria aplicaran el principio de precaución como parte de los principios generales de gestión de riesgo a lo largo del ciclo de vida de los nanomateriales manufacturados, entre otras 21 recomendaciones (IPEN, 2011). La resolución II/4 E de la ICCM2 invitó a preparar un informe sobre NT y productos nanofacturados considerando los temas relevantes para los países en desarrollo y con economías en transición. Para tal fin se han desarrollado talleres organizados por UNITAR (United Nations Institute for Training and Research) y la Organización para la Cooperación y Desarrollo Económico (OCDE) y se ha discutido el tema de las NT en las reuniones regionales del SAICM en diversas partes del mundo, incluyendo dos en América Latina y el Caribe: una realizada en Kingston, Jamaica, en marzo del 2010 y otra en Panamá en junio del 2011.

En la tercera reunión regional de América Latina y el Caribe del SAICM, celebrada en Panamá del 2 al 3 de junio del 2011 se discutieron y aprobaron por consenso una serie de recomendaciones sobre nanomateriales. ${ }^{6}$ Esta reunión estuvo precedida por un taller sobre nanotecnología organizado por UNITAR, donde participaron expertos de la Red Latinoamericana sobre Nanotecnología y Sociedad (ReLANS), de la Fundacentro del Ministerio del Trabajo de Brasil, de la Red Internacional de Eliminación de Contaminantes Orgánicos Persistentes (IPEN), y del Gobierno Suizo.

En dicha reunión de Panamá, y con base en un texto propuesto por IPEN, los delegados gubernamentales de los ministerios de ambiente o salud, y de organizaciones ambientales, de protección de la salud y asociaciones científicas, adoptaron una resolución donde se reconoce que la producción y el uso de nanomateriales está aumentando rápidamente en muchos de los países de la región, a pesar de la falta de información adecuada sobre los riesgos para la salud humana y el medio ambiente y de la ausencia de un marco legal/reglamentario nacional o de un marco normativo global. También recomendaron fortalecer las capacidades de los países de la región para evaluar de manera eficaz los riesgos potenciales de los nanomateriales manufacturados, especialmente para los grupos vulnerables, como los niños, las gestantes y las personas mayores; propiciar programas y medidas sobre salud ocupacional, con la consulta de los trabajadores y sus representantes, y sobre salud pública y salud ambiental, con la participación de todos los grupos de interés involucrados. Los participantes llamaron a establecer un comité multisectorial de expertos, encargado de proseguir la investigación sobre los nanomateriales manufacturados, a fin de reducir al mínimo los riesgos para la salud pública y ocupacional y para el medio ambiente. Tal resolución adoptada en Panamá convocó a exigir que los productores en- 
treguen información adecuada sobre el contenido de los nanomateriales manufacturados, a fin de dar a conocer los riesgos potenciales a las autoridades y a los consumidores a través del registro y etiquetado de los productos nano-manufacturados.

La resolución apoyó la propuesta realizada por el Gobierno Suizo de incorporar las NT como una nueva área del Plan Global de Acción del SAICM, e incluyó otras como la de promover el intercambio de información científica, técnica, legal e institucional para el desarrollo de capacidades que permitan una adecuada gestión de los nanomateriales manufacturados y su integración en las políticas públicas y programas de gestión de los productos químicos; e incentivar la generación, intercambio y diseminación de datos sobre la peligrosidad y los riesgos relacionados con los nanomateriales manufacturados.

Además, la resolución adoptada en Panamá solicitó que se prepare una resolución sobre NT y nanomateriales manufacturados para ser presentada a la ICCM-3, incluyendo recomendaciones para:

- El desarrollo de evaluaciones nacionales de los nanomateriales manufacturados y de instrumentos de orientación y capacitación basados en un enfoque de precaución, que puedan ser utilizados en los países por los gobiernos y por las partes interesadas;

- La elaboración de un marco normativo basado en un enfoque de precaución en relación a la salud pública, la salud ocupacional y el medio ambiente, a lo largo del ciclo de vida de los nanomateriales manufacturados;

- La inclusión de actividades relacionadas con las NT y nanomateriales manufacturados en los futuros acuerdos de financiación para el SAICM;

- El desarrollo de códigos aduaneros específicos para los nanomateriales manufacturados y de sistemas de clasificación internacionales armonizados;

- La elaboración de recomendaciones técnicas específicas para la eliminación ambientalmente adecuada de los residuos de nanomateriales manufacturados, con participación del Convenio de Basilea en lo que respecta a su peligrosidad;

- La aplicación de la responsabilidad ampliada del productor a través de todo el ciclo de vida de los nanomateriales manufacturados.

La resolución de América Latina y el Caribe, de Panamá, coincide con muchos puntos planteados por las resoluciones del Grupo de los países de África, y de Europa del Este y deberán ser consideradas por el informe que el Secretariado del SAICM debe elaborar sobre el tema en la Primera Reunión del Grupo de Composición Abierta, que es el órgano subsidiario que deberá preparar los proyectos de resolución sobre SAICM a ser aprobados en la tercera conferencia del ICCM en mayo-junio del 2012. Los países en desarrollo y con economías en transición tendrán que negociar estas propuestas con los intereses de países como Estados Unidos, Europa y Japón, 
que dominan la discusión de la OCDE, y discutir la propuesta del gobierno Suizo para incorporar este tema en el Plan Global de Acción del SAICM, donde seguramente se presentarán las demandas de asistencia técnica y financiera como exigencia para llegar a acuerdos.

Una de las debilidades del SAICM es su carácter voluntario; sin embargo, los países que son parte de este acuerdo tienen el compromiso de elaborar, actualizar y llevar a la práctica un plan nacional de implementación sobre una base interministerial y mediante un proceso abierto a la participación de todos los sectores sociales interesados. El Secretariado del SAICM está a cargo de la sección química de la División de Tecnología, Industria y Economía del Programa de Naciones Unidas para el Medio Ambiente (PNUMA), pero su aplicación debe involucrar los otros organismos que se relacionan con el ciclo de vida de los productos químicos, y ahora de los nanomateriales manufacturados, particularmente la Organización Mundial de la Salud.

Las organizaciones de la sociedad civil (científicos, ambientalistas, trabajadores y consumidores) tienen ante sí el reto de exigir ante las autoridades nacionales designadas que se cumplan los compromisos asumidos en el SAICM para una mayor protección a la salud y el ambiente y que se establezcan los mecanismos que permitan una participación plural de todos los sectores interesados en discutir las políticas que van a regular las NT, de modo de lograr una adecuada gestión con supervisión social. El rol de las organizaciones de la sociedad civil es esencial para evitar que se continúen introduciendo en el mercado productos con nanomateriales y nanopartículas cuyos riesgos a la salud y el ambiente no se hayan evaluado en forma suficiente. Las resoluciones de las reuniones regionales de SAICM y los próximos encuentros serán de gran importancia, ya que la $3^{a}$. Conferencia Internacional sobre la Gestión de los Productos Químicos, realizada en septiembre de 2012 en Nairobi, aceptó incluir a los nanomateriales manufacturados como tema del Plan de Acción Global de SAICM.

\section{Riesgos, sectores sociales involucrados y desarrollo responsable de las nanotecnologías: un enfoque a partir del ciclo de vida de las nanopartículas}

¿Cómo agregar, estimular y legitimar la participación de diversos sectores sociales en la discusión sobre la regulación y gestión del riesgo de las NT? Proponemos aquí un enfoque a partir del ciclo de vida de las nanopartículas. Este enfoque permite identificar diferentes grupos sociales expuestos a nanopartículas según el lugar que ocupan en las fases del ciclo de vida.

Por ciclo de vida de las nanopartículas se entiende el proceso que va desde la investigación, pasando por la producción e incorporación o aso- 
ciación de las nanopartículas a otras estructuras y la agregación de éstas en los productos de consumo final, hasta su conversión en desecho, una vez que dejan de cumplir la función para la cual fueron creadas, ya sea que se reciclen algunas de sus partes o que se descarten y salgan del circuito mercantil. Así, el ciclo de vida incluye:

Investigación y Desarrollo $\rightarrow$ Producción $\rightarrow$ Consumo $\rightarrow$ Desecho final

Cada etapa implica diferentes sujetos y condiciones de exposición a las nanopartículas. La investigación en NT se realiza en laboratorios, con determinados instrumentos según el tipo de partícula que se trabaje. Los principales sujetos de riesgo son los investigadores; y las rutas por las cuales las nanopartículas pueden interactuar con el organismo pueden ser varias, siendo la inhalación la más importante.

La producción de nano materiales en gran escala se realiza en instalaciones industriales, y las vías de interacción con el organismo de los trabajadores que las operan pueden ser similares a las anteriores. La producción también incluye la incorporación de nanopartículas, nanoestructuras o nanodispositivos a una gran variedad de productos de consumo final, como vestimenta, electrónicos, automóviles, construcción, cosméticos. Dependiendo del tipo de nanopartícula utilizada, de su incorporación al producto final y de la forma de consumo, los individuos que están expuestos a las nanopartículas varían, al igual que la potencial ruta de acceso al organismo, que bien puede ser la penetración por contacto con la piel en el caso de cosméticos, la inhalación cuando el producto desprende polvo durante su uso, la ingestión cuando se trata de un producto de la industria alimenticia o derivada y hasta la incorporación directa al flujo sanguíneo cuando ocurren accidentes.

Además de los riesgos a la salud humana, cada etapa puede implicar la liberación a la atmósfera, cursos de agua o suelo, de nanopartículas, lo que puede ocurrir inclusive de forma menos controlada cuando los productos se desechan y, en cada caso, hay riesgos particulares a los ecosistemas y seres vivos con los que interactúen.

Schulte, investigador del NIOSH y colaboradores, elaboraron el siguiente diagrama (Figura 3) del riesgo de exposición a nanopartículas en el lugar de trabajo. El diagrama excluye la incorporación de nanopartículas a otros productos finales, y también la fase de desecho de los productos. 
Figura 3

Locales de trabajo y potencial exposición a nanopartículas

\section{INVESTIGACIÓN}

Desarollo del concepto

Diseño de la molécula/particula

Identificación del mercado/aplicaciones

\section{Laboratorio de descubrimiento}

*Creación de cantidades para investigación: mg a gramos

*Optimización de rendimiento, prueba de materiales

Grupos expuestos: Investiga dores, técnicos, personal de mantenimiento y manejo de desechos

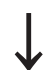

Laboratorio de ampliación de escala

*Creación de lotes en cantidade: $\mathrm{kg}$

*Prueba de material

*Desarollo de conceptos y clientes

Grupos expuestos: Investiga dores, técnicos, personal de mantenimiento, de manejo de desechos y transportistas

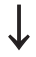

DESAROLLO

Proceso de diseño Producción/prueba de cantidades

Manejo interno y almacenamiento $\longrightarrow$ transporte

\section{Desarollo de procesos}

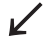

*Optimización del proceso de diseño

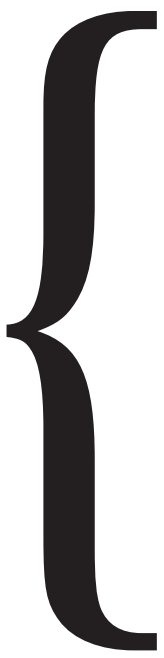

*Conducción de apliación de escala

*Prueba de producto en cantidade: $\mathrm{kg}$ a cientos de $\mathrm{kg}$

Grupos expuestos: Investiga dores y prácticos, técnicos,

personal de prueba, mantenimiento, desecho y transporte<smiles>C[AlH]</smiles>

Almacenamiento interno y externo $\longrightarrow$ transporte

Desarollo de productos

* Prueba de cantidad de mercado: cientos de kg

*Ampliación de aplicaciones a consumidores

$\longleftarrow$ Materia prima e intermedia $\longrightarrow$ Desecho

*Optimización de procesos y del flujoo material: "hacer, empacar, transportar"

Grupos expuestos: Investiga dores y prácticos, técnicos, personal de proceso y mantenimiento de facilidades, alma cenamiento, transporte y manejo de desechos

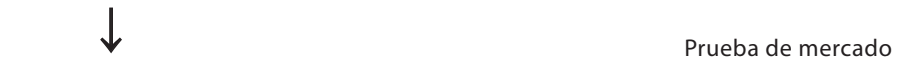

Material producido $\longrightarrow$ Empaque $\longrightarrow$ Transporte

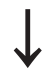

Operación \& Producción sostenida

Grupos expuestos: Técnicos de producción, personal de investiga ción y desarrollo, de processos y mantenimiento de facilidades, almacenamiento y transporte

Pruebas experimentales

Desarrollo de aplicadores
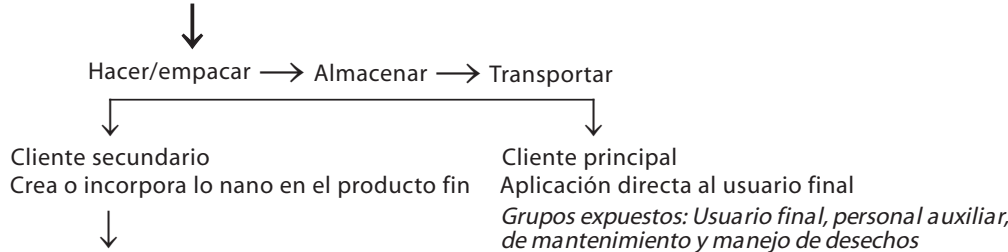

Desarrollo de producto

Prueba de mercado

Formulación

Aplicación

Producción $\longleftarrow$ Materia prima e intermedia

Fuente: DHHS, p. 38, 2009.

Nota: La figura ilustra el ciclo de vida de los nanomateriales desde el laboratorio de investigación y desarollo a través de la evolución del producto, uso y disposición final. Cada etapa del ciclo representa posibilidades de exposición de los trabajadores a nanoparticulas. 
El diagrama se centra en el riesgo de exposición laboral en el ciclo de vida de las nanopartículas manufacturadas. Por esta razón no aparecen los riesgos al medio ambiente, y tampoco los riesgos a los consumidores, pero dando seguimiento a cada etapa resulta fácil intuirlos. Así, por ejemplo, todas las etapas muestran, en la sección de la derecha, que existen residuos y éstos pueden afectar el medio ambiente si no existieran mecanismos adecuados para evitarlo.

El diagrama también muestra que, para cada etapa, existen sujetos de riesgo diferentes. Es claro que en la etapa de investigación los propios investigadores son sujeto de riesgo; pero los autores advierten que también lo son los trabajadores de servicio de mantenimiento de los laboratorios, y también son proclives al riesgo quienes se encargan de manipular los desechos.

La segunda etapa que se ilustra en el diagrama es la de desarrollo o producción de materias primas en masa. Aquí los individuos directamente involucrados son investigadores, técnicos y operarios de las empresas, aunque se agregan los encargados de almacenar el producto y los transportistas que llevan la nano-materia prima a las industrias que las requieren.

Luego viene la etapa de la producción y manufactura de productos intermediarios y finales. Aquí las nanopartículas son incorporadas a variados procesos industriales, con el fin de otorgar a los productos una ventaja comercial o de utilidad. Además de los sujetos de riesgo de las etapas anteriores surgen diversos sectores de obreros y personal administrativo y de servicios.

Aunque en el diagrama constituye una subdivisión de la etapa de producción y manufactura, el consumidor final es, en realidad, un sujeto específico en la cadena de exposición a nanopartículas.

Debe quedar claro que la conexión entre exposición a nanopartículas y riesgo a la salud está mediada por múltiples factores, no solamente los que son intrínsecos a los propios nanomateriales y grado de exposición de los trabajadores, sino también, y de forma muy importante, las condiciones de infraestructura, equipamiento de supervisión, instrumentos y vestimenta de protección y los medios de prevención de aquellas exposiciones.

A pesar de la generalidad de la explicación del diagrama y su reducción a grandes etapas, si algo queda claro es que son los trabajadores quienes están en primera instancia sujetos a la exposición y, consecuentemente, al potencial riesgo de las nanopartículas. En muchos de los productos finales, las nanopartículas están incorporadas a una matriz, que hace difícil su desprendimiento e interconexión con el organismo biológico. Sin embargo, los trabajadores que fabrican nanopartículas, o los trabajadores de una industria textil o de otro sector que incorporan nanopartículas a sus tejidos o producto final, están expuestos a las nanopartículas libres, aún no asociadas de manera físicoquímica al producto final y, por tanto, su grado de exposición es mayor que la del consumidor final. Existen, sin embargo, casos en que el consu- 
midor final puede estar directamente expuesto, como ocurre con los cosméticos, donde la nano-materia prima no está asociada a una matriz, sino libre en la forma de polvo en el producto, pudiendo entonces introducirse con mayor facilidad en el organismo. Otro ejemplo es el caso de los trabajadores agrícolas que manipulan plaguicidas y otras sustancias químicas directamente, y donde un mínimo accidente puede implicar el contacto directo del producto que contiene las nanopartículas con la piel, la inhalación de vapores u otras formas de exposición.

Desde el punto de vista de la actividad de SAICM, que se preocupa por definir acciones e incorporar los diversos sectores de la sociedad a tales acciones, la identificación de los sindicatos como representantes de los trabajadores y de ONG como representantes de los consumidores es crucial para muchas de las actividades, ya que permite conectar más claramente una propuesta de actividad con el sector social más proclive a sufrir las consecuencias de un mal manejo de los productos que contienen nanopartículas. Desde otro punto de vista, también es importante la participación de los industriales que producen nanomateriales y nanodispositivos y los que los usan en productos de consumo final, ya que son los responsables directos de eventuales exposiciones y riesgos. De la misma manera, los diferentes niveles de la administración pública tienen su responsabilidad en la supervisión y reglamentación de las actividades, razón por la cual el papel de representantes del Estado es esencial en las discusiones colectivas. Además, la preocupación con el potencial riesgo sobre el medio ambiente requiere la participación de las organizaciones sociales que se preocupan con esta dimensión.

Si el ciclo de vida permite identificar sectores sociales específicos como interlocutores necesarios, hay tres tipos de acciones que son requisito para un desarrollo responsable de las NT. En primer lugar, la investigación y transparencia en la información. Es de interés de los diferentes sectores que existan suficientes fondos públicos para investigar los potenciales riesgos de las NT, evitando que la dinámica del mercado genere riesgos y accidentes. Pero también es importante que los sindicatos, como representantes de los trabajadores, sepan si la empresa en la que se desempeñan está de alguna manera trabajando con nanomateriales. Como medida de precaución algunos sindicatos podrían negociar la incorporación a los contratos colectivos de trabajo de cláusulas que obliguen a la patronal a informar cuando incorporen NT a sus procesos. La Organización Internacional del Trabajo, en su Convenio 154 sobre negociación colectiva respalda que se adopten medidas para que las partes dispongan de la información necesaria.

El segundo tipo de acción se refiere a la regulación gubernamental. Los gobiernos deben velar por la salud de los trabajadores, consumidores y medio ambiente, y para ello la regulación de los procesos de NT constituye 
un aspecto central. A falta de información suficiente el principio de precaución debiera ser la guía.

Hay un tercer tipo de acción que se ha venido implementando: las certificaciones voluntarias, o los códigos de conducta voluntarios. Estos son declaraciones de empresas y asociaciones de empresas, o inclusive de gobiernos dirigidas al consumidor, con el propósito de publicitar un determinado comportamiento responsable en los procesos productivos. Aunque se trata de medidas proactivas, su implementación como medida voluntaria no es garantía para la mayoría de la población. Es por esta razón que a mediados del 2007 más de cien organizaciones no gubernamentales, sindicatos y otras organizaciones civiles lanzaron la declaración llamada Principios para la Supervisión de las Nanotecnologías y Nanomateriales (NanoAction, 2007). Allí se argumenta y justifica a favor de que las políticas públicas reconozcan y/o adopten ocho principios respecto de los productos nanomanufacturados y las NT.

Cuadro 1

Principios para la Supervisión de las Nanotecnologías y Nanomateriales

1. Principio de precaución. Cuando exista duda razonable de que productos o procesos pueden significar una amenaza para la salud humana o el medio ambiente debe aplicarse la precaución, aún cuando no exista comprobación científica contundente. La comprobada alta reactividad y movilidad, entre otras propiedades de las nanopartículas, pueden generar toxicidad desconocida, lo cual justifica aplicar el principio de precaución.

2. Principio sobre las regulaciones obligatorias específicas para los nanomateriales. Dado que los materiales manufacturados en nanoescala manifiestan propiedades físicas, químicas y biológicas diferentes a las que se conocen en tamaños mayores, deben ser clasificados como nuevas substancias para cualquier fin regulatorio. La actual legislación basada en tamaños mayores, y por tanto propiedades físicas diferentes, es inadecuada para regular los nanomateriales; debe elaborarse una reglamentación específica. Las iniciativas voluntarias han demostrado ser insuficientes para cualquier tipo de regulación.

3. Principio de protección y salud del público y los trabajadores. Los trabajadores de la nano-industria están sujetos a las mayores exposiciones y, por tanto, a amenazas muchas veces desconocidas. Los consumidores pueden estar expuestos a las nanopartículas mediante los productos que las contienen, pero también por causa del destino final de los nanoproductos como desechos, por la liberación al medio ambiente de nanopartículas y por el reciclado de productos. Estas razones son más que suficientes para otorgar énfasis inmediato a una política preventiva en tales ámbitos.

4. Principio sobre la sustentabilidad ambiental. Existen suficientes elementos que demuestran que muchos nanomateriales no se biodegradan fácilmente, por lo cual su ciclo de vida (investigación y desarrollo, manufactura, transporte y almacenamiento, uso y consumo, reciclado y desecho) puede llevarlos a ser acumulados en ecosistemas y otros seres vivos con consecuencias perjudiciales. Es necesario que el ciclo de vida completo de los nanomateriales, nanoestructuras y su asociación con productos finales sea analizado antes de su comercialización.

5. Principio de transparencia. El público tiene el derecho de conocer lo que consume; para ello es necesario que todos los productos de la nanotecnología sean etiquetados. Además, los trabajadores de las nano-industrias deben disponer de la información necesaria que les permita actuar conforme a su seguridad. 
6. Principio de participación pública. La participación pública en los procesos deliberativos es un elemento esencial de la vida democrática. Todos los actores e interesados deben poseer la información necesaria para poder actuar en defensa de sus derechos e intereses. La participación del público debe contribuir a la formación de políticas y toma de decisiones, en lugar de ser solamente un consumidor de eventos en donde el gobierno y las industrias deciden unilateralmente.

7. Principio sobre la consideración de amplios impactos. Las nanopartículas manufacturadas no solamente presentan riesgos a la salud humana y el medio ambiente. Existe una amplia gama de diversas implicaciones sociales. La industria de la nanotecnología puede arruinar muchas otras industrias, provocando problemas de empleo, de comercio y de inseguridad social; y representa retos nuevos en términos legales. Las nanotecnologías tienen el potencial de alterar biológicamente al ser humano y de crear nuevos seres vivos, generando implicaciones éticas. El debate público de todos estos aspectos es relevante para un desarrollo que considere los intereses y posiciones de los diversos sectores de la sociedad.

8. Principio de responsabilidad del fabricante. La falta de investigación de varios productos lanzados al mercado ha provocado impactos innecesarios a la salud y el medio ambiente, como fue el caso del asbesto. Hoy en día existen las condiciones científicas y técnicas para que los nanomateriales sean profundamente analizados antes que se vendan al público y generen peligros innecesarios. Para ello es imperioso que quienes desarrollen, produzcan y comercialicen productos de la nanotecnología se hagan responsables de los daños causados por dichos productos a lo largo de su ciclo de vida.

Fuente: Principios para la supervision de las nanotecnologías y nanomateriales (Nanoaction, 2007).

Como muchos de los productos que contienen nanopartículas están ingresando al mercado de los países de América Latina y el Caribe como importaciones, el incipiente desarrollo nacional y regional de productos con NT no es razón para que los países de América Latina y el Caribe no se preocupen por tomar actitudes proactivas al respecto.

El SAICM representa un espacio de negociación multilateral que permite la participación de los diferentes sectores de la sociedad. Es importante que estas negociaciones tengan en cuenta que los diferentes sectores de la sociedad enfrentan diversos grados de riesgo según su relación con las fases del ciclo de vida de los nano-materiales manufacturados y su exposición. Los trabajadores de la nano-industria, incluyendo la investigación, producción, almacenamiento, transporte y desecho, así como los trabajadores de industrias que incorporan nano materiales como materia prima de otros procesos mayores, y los consumidores son los más susceptibles de estar expuestos a los riesgos de las nanotecnologías; y el medio ambiente se puede ver afectado tanto por la liberación incontrolada de nanopartículas como por el destino final de los productos que las contienen. Estas razones obligan a una discusión ordenada de las necesidades y características de cada país y de los diversos sectores involucrados para reducir o eliminar los riesgos de las nanotecnologías a la salud y el medio ambiente y lograr así los objetivos del SAICM. 


\section{Notas}

1 Profesor del Doctorado en Estudios del Desarrollo de la Universidad Autónoma de Zacatecas, México. Miembro de ReLANS (Red Latinoamericana de Nanotecnología y Sociedad). Doctor en economía por la Universidad Nacional Autónoma de México. <gfoladori@gmail.com> Correspondência: Sierra Madre Occidental 101 B. Zacatecas, Zac 98600. México.

2 Sociólogo, punto de enlace de IPEN (International Persistent Organic Pollutants Elimination Network) para América Latina y director del Centro de Análisis y Acción en Tóxicos y sus Alternativas (CAATA) en México. Maestro en Ciencias por COLPOX, México. $<$ coordinacion@caata.org.mx >

3 Profesora del Sector de Educación y del Programa de Pós-Graduación en Políticas Públicas de la Universidad Federal de Paraná (UFPA). Miembro de ReLANS (Red Latinoamericana de Nanotecnología y Sociedad). Doctora en Política Científica y Tecnológica por la Universidade Estadual de Campinas, Brasil. <noela.invernizzi@gmail.com>

4 De 2004 a 2008 fueron invertidos 233.9 millones de reales. El dato disponible para 2009 es estimado, y corresponde a 80 millones de reales.

${ }^{5}$ La meta del 2020 fue establecida en el párrafo 23 del Plan de Implementación de la Cumbre Mundial sobre Desarrollo Sostenible realizada en Johannesburgo, Sudáfrica, en el 2002 , en la sección sobre "la gestión racional de las sustancias químicas", en el contexto de "cambiar los modos de producción y consumo no sostenibles."

6 Sólo en las dos últimas reuniones (Kingston 2010 y Panamá 2011) se trató expresamente el tema de los nanomateriales manufacturados.

\section{Referências}

CHIANCONE, Adriana. Nanociencia y Nanotecnologías en Uruguay: áreas estratégicas y temáticas grupales. In: FOLADORI, Guillermo; ZÁYAGO, Edgar; INVERNIZZI, Noela. Perspectivas sobre el desarrollo de las nanotecnologias en América Latina. México DF: Miguel Angel Porrúa, (en prensa). Cap. 10.

\section{CIMAV (CENTRO DE INVESTIGACIONES} EN MATERIALES AVANZADOS) (México). Diagnóstico y prospectiva de la nanotecnología en México. Chihuahua: Secretaría de Economía/Cimav, 2008. 130 p. Disponible en: $<$ www.nanotech.cimav.edu.mx $>$. Acceso en: 14 ene. 2011.
CNNN (NATIONAL COUNCIL FOR NANOSCIENCE AND NANOTECHNOLOGY). 2005, Bogotá. CONFERENCIA INTERNACIONAL, NANOFORUM COLOMBIA. Bogotá: Ieee.org, 2005. Disponible en: <www.nanocolombia. org $>$ / <nanocolombia@ieee.org > . Acceso en: 10 out. 2005.

CONACYT (CONSEJO NACIONAL DE CIENCIA Y TECNOLOGÍA) (México). Programa especial de ciencia y tecnología 2001-2006. [Véase también tomo II]. México DF: Conacyt, 2002.

CORTÉS-LOBOS, Rodrigo. Nanotecnología en Chile, ¿Qué tan preparado se encuentra 
el país para desarrollar esta disciplina? In: FOLADORI, Guillermo; ZÁYAGO, Edgar; INVERNIZZI, Noela. Perspectivas sobre el desarrollo de las nanotecnologías en América Latina. México DF: Miguel Angel Porrúa, 2011. (en prensa). Cap. 4.

CUBADEBATE, Cuba. Culmina en Cuba seminario internacional de nanotecnologías. CubaDebate, La Habana, 09 set. 2010. Disponible en: <www.cubadebate.cu/noticias/ 2010/09/09/culmina-en-cuba-seminariointernacional-de-nanotecnologías/>. Acceso en: 02 mayo 2011

DHHS (DEPARTMENT OF HEALTH AND HUMAN SERVICES). Centers for Disease Control and Prevention. National Institute for Occupational Safety and Health (Estados Unidos). Approaches to Safe Nanotechnology, DHHS NIOSH Publication No. 2009-125, 2009.

Centers for Disease Control and Prevention. National Institute for Occupational Safety and Health (Estados Unidos). Approaches to Safe Nanotechnology, DHHS NIOSH Publication No. 2009-125, p. 38, 2009. Disponible en: $<$ www.cdc.gov/niosh/docs/2009-125/pdfs/ 2009-125.pdf > . Acceso en: 4 feb. 2013.

EMBRAPA NOTÍCIAS. Coordenador do MCT anuncia recursos de R\$ 1 milhão para popularização da nanotecnologia, 14/09/2009. Disponible en: <www.embrapa.br/imprensa/noticias/ 2009/setembro/3a-semana/coordenador-domct-anuncia-rercursos-de-r-1-milhao-parapopularizacao-da-nanotecnologia $>$. Acceso en: 14 feb. 2011.

FOLADORI, Guillermo; Záyago, Edgar. La política de Ciencia y Tecnología en México y la incorporación de las nanotecnologías. In: FOLADORI, Guillermo; ZÁYAGO, Edgar; INVERNIZZI, Noela. Perspectivas sobre el desarrollo de las nanotecnologías en América Latina. México DF: Miguel Angel Porrúa, (en prensa). Cap. 7.

FONARSEC (Argentina). Bases de la convocatoria Fondo Sectorial de Nanotecnologia FSNano-2010. Buenos Aires: ANPCyTMINCyT, 2010.
GARCÍA, Marisa; LUGONES, Manuel; REISING, Ailin María. Conformación y desarrollo del campo nanotecnocientífico argentino: una aproximación al estado de la cuestión desde el estudio de los instrumentos de promoción científica y tecnológica. In: FOLADORI, Guillermo; ZÁYAGO, Edgar; INVERNIZZI, Noela. Perspectivas sobre el desarrollo de las nanotecnologías en América Latina. México DF: Miguel Angel Porrúa, (en prensa). Cap. 1.

GUTARRA, Abel. Estado de situación de la nanotecnología en el Perú. 2008. Disponible en: <www.nanotecnologia.com.pe/docs/ nanotecnologia.pdf $>$. Acceso en: 30 mar. 2011.

INVERNIZZI, Noela; KORBES, Cleci; FUCK, Marcos. Política de Nanotecnología en Brasil: a 10 años de las primeras redes. In: FOLADORI, Guillermo; ZÁYAGO, Edgar; INVERNIZZI, Noela. Perspectivas sobre el desarrollo de las nanotecnologías en América Latina. México DF: Miguel Angel Porrúa, (en prensa). Cap. 3.

IPEN (Red Internacional de Eliminación de Contaminantes Orgánicos Persistentes). Breves antecedentes sobre nanotecnología y nanomateriales. 2011. Disponible en: <www.ipen. org/ipenweb/work/nano.html>. Acceso en: 30 mar. 2011

KAY, Luciano; SHAPIRA, Paul. Developing Nanotechnology in Latin America. Journal of Nanoparticle Research, New York, vol. 11, p. 259-278. 2009.

KULINOWSKI, Kristen. Temptation, Temptation, Temptation: Why Easy Answers About Nanomaterial Risk are Probably Wrong. AzoNanotechnology. 2009.

LÓPEZ, María Sonsiré ; HASMY, Anwar; VESSURI, Hebe (2011) Nanociencia y Nanotecnología en Venezuela. In: FOLADORI, Guillermo; ZÁYAGO, Edgar; INVERNIZZI, Noela. Perspectivas sobre el desarrollo de las nanotecnologías en América Latina. México DF: Miguel Angel Porrúa, (en prensa). Cap. 11.

MAYNARD, Andrew D. Nanotechnology: Assessing the risks. NanoToday, Cambridge, MA, vol. May. 22-33. 2006. 
MAYNARD, Andrew D., et al. Safe handling of nanotechnology. Nature, London, v. 444, p. 6, 2006.

MCT (Ministério da Ciência e da Tecnologia). Relatório analítico Programa de C,T\&I para Nanotecnologia. Coordenação Geral de Micro e Nanotecnologias, Brasília, 2008. Disponible en: <www.mct.gov.br/upd_blob/ 0028/28213.pdf>. Acceso en: 22 feb. 2011.

MEDINA Vázquez, Javier (2005). Investigación y Desarrollo Tecnológico en Colombia. Disponible en: <http://les.man.ac.uk/PREST/ SCOPE/documents/National_Report_Colombia. pdf>. Acceso en: 20 oct. 2005.

NANOACTION (2007). Principios para la supervision de las nanotecnologías y nanomateriales. NanoAction. A Project of the International Center for Technology Assessment. 2007. Disponible en: <www.nanoaction.org/ nanoaction/page.cfm?id=223>. Acceso en 17 jun. 2011.

NANOCEO (Nanotechnology Citizen Engagement Organization). Database. Disponible en: $<$ www.nanoceo.net/nxanorisks $>$. Acceso en: 20 dic. 2010.

OBERDORSTER, Gunter; OBERDORSTER, Eva; OBERDORSTER, Jon. Nanotoxicology: An Emerging Discipline Evolving from Studies of Ultrafine Particles. Environmental Health Perspectives, Bethesda, MD, p. 259278. 2005.

OICTI (OBSERVATORIO IBEROAMERICANO DE CIENCIA TECNOLOGÍA E INNOVACIÓN). La Nanotecnología en Iberoamérica. Situación Actual y Tendencias. Observatorio Iberoamericano de Ciencia, Tecnología e Innovación Del Centro de Altos Estudios Universitarios de La Organización de Estados Iberoamericanos, 2009. 444 p. Disponible en: <www.oei. es/observatoriocts/index.php?option $=\mathrm{com}$ content\&view $=$ article\&id $=12 \&$ Itemid $=3>$. Acceso en: 16 mar. 2011.

PIAZZA, Fabrice (2011) Nanociencias y Nanotecnologías en la República Dominicana. In: FOLADORI, Guillermo; ZÁYAGO,
Edgar; INVERNIZZI, Noela. Perspectivas sobre el desarrollo de las nanotecnologias en América Latina. México DF: Miguel Angel Porrúa, (en prensa). Cap. 09.

RS\&RAE (Royal Society and Royal Academy of Engineering. NANOSCIENCE AND NANOTECHNOLOGIES: OPPORTUNITIES AND UNCERTAINTIES (Policy document 20/04), 2004. London: The Royal Society and the Royal Academy of Engineering.

SINACYT/CONCYTEC (Science, Technology and Innovation National System/National Council for Science and Technology). Plan Nacional Estratégico de Ciencia, Tecnología e Innovación para la Competitividad y el Desarrollo Humano (PNCTI) 2006-2021, 2005. Lima: Concytec.

SPIVACK, Ana; et al. La estructuración de la investigación argentina en nanociencia y nanotecnología: balances y perspectivas. In: FOLADORI, Guillermo; ZÁYAGO, Edgar; INVERNIZZI, Noela. Perspectivas sobre el desarrollo de las nanotecnologías en América Latina. México DF: Miguel Angel Porrúa, (en prensa). Cap. 2.

TOMA, H. E. Interfaces e organização da pesquisa no Brasil: da Química à Nanotecnologia. Química Nova, v. 28. p. 48-51. 2005. Disponible en: <www.scielo.br/pdf/qn/v28s0/26775.pdf>. Acceso en: 16 mar. 2011.

VEGA-BAUDRIT, José R.; CAMPOS, Allan. Nanotecnología en la región centroamericana y Panamá: caso Costa Rica. In: FOLADORI, Guillermo; ZÁYAGO, Edgar; INVERNIZZI, Noela. Perspectivas sobre el desarrollo de las nanotecnologías en América Latina. México DF: Miguel Angel Porrúa, (en prensa). Cap. 4.

WEINBERG, Jack. Una Guía al SAICM para las ONGs. Red Internacional de Eliminación de los contaminantes Orgánicos Persistentes (IPEN). 2008. Disponible en: <www.ipen. org >. Acceso en: 16 mar. 2011.

Recebido em 06/07/2011

Aprovado em 21/12/2011 